and the L/P ratio will not increase. ${ }^{2}$ Some drugs, such as oral hypoglycemics, ethanes, catecholamines, and beta- 2 bronchodilators, elevate blood lactate levels without causing tissue hypoxia. There are probably two types of hyperlactatemia in stable patients with a very different prognostic significance: hyperlactatemia secondary to aerobic overproduction, with a good prognosis, or a "true" hyperlactatemia caused by the inadequate use or insufficient clearance of lactate, with a poor prognosis. As demonstrated by Dr. Koliski in her study, high lactate level was initially a good predictor of death, but only in the initial phase of instability, during the first 24 hours, and not later, when higher stability was achieved. This fact concurs with that which was published by Levy et al. ${ }^{3}$ where initial lactate levels were not different between survivors and those who died after 24 hours. De Backer \& Creteur ${ }^{4}$ reported that hyperlactatemia in septic adults was equivalent between survivors and those who died immediately after stabilization. It has also been described that septic patients with normal lactate levels may or may not develop complications, that is, normal lactate levels may not have a prognostic value. Another point of view is that some septic patients show normal lactate levels because the low production of lactate is offset by its poor clearance.

Despite the fact that the study carried out by Dr. Koliski investigated a heterogeneous population, it revealed that initially high lactate levels are an important marker for severity. In some situations, they may be more specific than usual macrohemodynamic measurements. ${ }^{5}$

\section{References}

1. Koliski A, Cat I, Giraldi DJ, Cat ML. Lactato sérico como marcador prognóstico em crianças gravemente doentes. J Pediatr (Rio J). 2005;81:287-92.

2. Mizock BA. Redox pairs, tissue hypoxia, organ dysfunction and mortality. Crit Care Med. 2000;28:270-2.

3. Levy B, Saudone L. Evolution of lactate/pyruvate and ketone body ratios in the early course of catecholamine-treated septic shock. Crit Care Med. 2000;28:114-19.

4. De Backer D, Creteur J. The hepatoesplachnic area is not a common source of lactate in patients with severe sepsis. Crit Care Med. 2001;29:256-1.

5. Ronco R, Rodríguez J, Castillo A. Intragastric tonometry outcome predictor and monitoring utility in pediatric shock [abstract]. Pediatr Chil J. 1998;69:60-4.

\title{
Continuous glucose monitoring: a practice that should be explored
}

\author{
Durval Damiani *
}

D guarantee the integrity of several tissues, organs and systems of the human body. However, it was only in 1993, with the conclusion of a large research study with diabetic patients, known as the Diabetes Control and Complications Trial (DCCT), ${ }^{1}$ that it became clear that glucose control in diabetes mellitus (DM) was one of the key factors to avoid medium- and long-term complications.

Several insulin delivery systems were developed in an attempt to mimic what the body usually does, that is, to deliver insulin every time we eat something. The fact that this should occur at the right place (pancreatic islets that

* Full professor, Pediatric Endocrinology Unit, Instituto da Criança do Hospital das Clínicas, Universidade de São Paulo (USP), São Paulo, SP, Brazil.

Suggested citation: Damiani D. Continuous glucose monitoring: a practice that should be explored. J Pediatr (Rio J). 2005;81:272-3. provide insulin to the liver first), at the right time and at the right amount, makes the attempt to reproduce in diabetic patients what occurs in nondiabetic ones a reasonably complicated task. In a continuous monitoring study with healthy volunteers, glucose levels oscillated from 46 to 118 , showing how masterful the glucose control system has to be in order to maintain an appropriate "metabolic environment" and to avoid complications caused by protein glycation. ${ }^{2}$ Today, we use the so-called intensive management of DM, a method that aims to maintain patients with insulin administration during 24 hours, using higher doses of ultrafast insulin at meal times. However, intensive management is synonymous with intensive control of glucose levels, unless we decide to "fly without instruments!"

Monitoring methods have been improved, requiring smaller amounts of blood and delivering results within a shorter time. There are noninvasive methods, which determine interstitial glucose levels, and sensors that allow continuous monitoring for a given period of time. This 
method is known as the Continuous Glucose Monitoring System (CGMS).

In patients' daily routine, glucose meters perform their role properly, but some questions should be raised, given the association between monitoring frequency and metabolic control: ${ }^{3}$ when should glucose levels be measured? Before or after meals? How long after meals? Before and after meals? How many pricks would be "acceptable" considering pricking sites (fingertip and others) and also economic implications? We should not forget that test strips are quite expensive.

In 1999, the FDA approved the glucose sensor, a pagersized monitor attached to a subcutaneous sensor that provides glucose measurements every 10 seconds, with an average of 60 readings, plotting one point every five minutes. Usually, the device remains implanted for 72 hours. However, the information is not given to the patient, but later analyzed by a computer program. Its objective is to evaluate glucose excursions during 24 hours, on three consecutive days, and to check whether the results are consistent from one day to the next, and at what level and at what time glucose oscillations preferably occur. These data help fine-tune the methods for insulin delivery, delivery times, dietary patterns, physical exercise, etc. It is certainly an auxiliary tool in the appropriate management of diabetic patients, but it has to be analyzed with "expert knowledge," so that the collected data can produce better glucose control.

The study of Maia \& Araújo, published in the current issue of Jornal de Pediatria, retrospectively evaluates 16 patients with type $1 \mathrm{DM}$, aged, on average, 16 years, who were submitted to glucose monitoring for 72 hours, during which period, the following parameters were analyzed: mean capillary blood glucose level obtained with the glucose meter and with the glucose sensor, glucose excursions, occurrence of postprandial hyperglycemia, nocturnal hypoglycemia, complications related to the use of the glucose sensor, and therapeutic management based on the data obtained from continuous monitoring. ${ }^{4}$ In my view, the most important conclusion drawn by the authors was that the therapeutic management was changed in $100 \%$ of the patients and that the "adjustment of therapeutic measures" caused a hemoglobin A1c decrease of nearly one percentage point after three months. This means a huge reduction in the risks of complication, since DCCT has shown that every time hemoglobin A1C decreases by one percentage point, ophthalmologic, renal and neurological complications decrease by 25 to $30 \%$. On the other hand, the detection of nocturnal hypoglycemia, which is most feared because its symptoms may not be perceived by sleeping patients, requires that the therapeutic management be changed in order to prevent neurological sequelae. In a study with 10 children under the age of six years with type 1 DM, CGMS revealed that $7 \%$ presented hypoglycemia (a level twice as high as the one detected with conventional monitoring) and that nocturnal hypoglycemic episodes lasted longer than daytime episodes. ${ }^{5}$ In a study of 109 patients, 51 of whom were submitted to CGMS and 58 to conventional monitoring, Tanenberg et al. did not find any differences between the levels of glycated hemoglobin after 12 hours in both groups, but the CGMS group showed a shorter duration of hypoglycemic episodes. This improvement in glucose control is highlighted by the authors. ${ }^{6}$

Deiss et al. submitted 50 patients to CGMS and managed to reduce $\mathrm{HbA} 1 \mathrm{c}$ from 8.1 to $7.7 \%$ in 6 weeks by changing the insulin administration practices. In patients who showed higher initial levels of $\mathrm{HbA} 1 \mathrm{c}(8.9 \%)$, the reduction amounted to $0.8 \%$ (from 8.9 to $8.1 \%)^{7}$

A great deal of effort should be put in to obtain glucose levels as close as possible to those of nondiabetic individuals. Special attention should be given to schemes that cause severe hypoglycemia (especially nocturnal hypoglycemia) and to hypoglycemia unawareness, in which the patient does not perceive the presence of hypoglycemia and, consequently, does not take the necessary precautions to reverse it, which leads to potential central nervous system involvement.

Continuous monitoring sensors open the way for the "smart insulin infusion pump," in which the information collected by the sensor is transmitted by telemetry (in the future, the sensor and the pump will be assembled into the same device), making the pump deliver the appropriate amount of insulin. This will be the so-called artificial endocrine pancreas, which will surely make the lives of diabetic patients a lot easier.

\section{References}

1. Diabetes Control and Complications Trial Research Group. Effect of intensive diabetes treatment on the development and progression of long-term complications in adolescents with insulin-dependent diabetes mellitus: Diabetes Control and Complications Trial. J Pediatr. 1994;125:177-88.

2. Ionescu-Tirgoviste C, Guja C, Ioacara S, Dumitrescu D, Tomescu I. Continuous glucose monitoring: physiologic and pathophysiologic significance. Rom J Intern Med. 2004;42: 381-93.

3. Bui H, Perlman K, Daneman D. Self-monitoring of blood glucose in children and teens with diabetes. Pediatr Diabetes. 2005;6: 50-62.

4. Maia FF, Araújo LR. Accuracy, utility and complications of continuous glucose monitoring system (CGMS) in pediatric patients with type 1 diabetes. J Pediatr (Rio J). 2005;81:293-7.

5. Jeha GS, Karaviti LP, Anderson B, Smith EO, Donaldson S, McGirk TS, et al. Continuous glucose monitoring and the reality of metabolic control in preschool children with type 1 diabetes. Diabetes Care. 2004;27:2881-6.

6. Tanenberg R, Bode B, Lane W, Levetan C, Mestman J, Harmel $A P$, et al. Use of the continuous glucose monitoring system to guide therapy in patients with insulin-treated diabetes: a randomized controlled trial. Mayo Clin Proc. 2004;79:1521-6.

7. Deiss D, Hartmann R, Hoeffe J, Kordonouri O. Assessment of glycemic control by continuous monitoring system in 50 children with type 1 diabetes starting on insulin pump therapy. Pediatr Diabetes. 2004;5:117-21. 